\title{
High stability in near-infrared spectroscopy: part 2, optomechanical analysis of an optical contacted V-shaped cavity
}

\author{
Nicolas Jobert ${ }^{1} \cdot$ Mathieu Casado $^{2,3,4}{ }^{\mathbb{D}} \cdot$ Samir Kassi ${ }^{2}$
}

Received: 27 April 2021 / Accepted: 7 December 2021 / Published online: 27 February 2022

(c) The Author(s) 2022

\begin{abstract}
Optical feedback is a powerful technique to stabilise and narrow semi-conductor lasers. As a step forward for field deployable, ultra-stable yet tunable sources, we analyse and model the opto-mechanical design of a V-shaped cavity optical feedback (VCOF) reference cavity. We estimate the relative contributions of sources of external disturbance on the optical resonance frequency of the cavity, and ultimately define the minimal thermal and mechanical shielding requirements to face field conditions. We attest of the robustness of the developed system, and show that external sources of disturbance are only secondary contributors to the overall stability of the cavity. The suite of analytical tools developed in the process paves the way for lighter and more compact cavity designs, more adapted to field deployment.
\end{abstract}

\section{Introduction}

Infrared spectroscopy is a commonly used technique for rapid and accurate trace detection measurements in gas $[1,2]$. In the case of isotopic composition, more stringent requirements are necessary [3-5], justifying more efforts to improve the detection performances. As the measurements of gas species rely on measuring the areas, it is crucial to have not only a good sensitivity, as provided by Cavity RingDown Spectroscopy (CRDS) techniques [6], but also a fine and stable frequency source [7]. The development of more powerful instrument (either in term of detectivity limit or of stability), is justified by the growing need for precise measurements: in the laboratories for fundamental physical properties determination $[8,9]$, and for field studies in geosciences $[10,11]$.

Optical Feedback [12-14], is a method which enables the transfer of the physical properties of an optical resonator

Mathieu Casado

mathieu.casado@gmail.com

1 ALMA Consulting, Arcueil, France

2 LiPHY, Laboratoire Interdisciplinaire de Physique, Université Grenoble Alpes/CNRS, Grenoble, France

3 Laboratoire des Sciences du Climat et de l'Environnement, LSCE/IPSL, CEA-CNRS-UVSQ, Université Paris-Saclay, Gif sur Yvette, France

4 Present Address: Alfred Wegener Institut Helmholtz Centre for Polar and Marine Research, Potsdam, Germany (thermal and mechanical stability as well as optical properties) to a laser diode $[12,15]$, and thus provides a solution to improve the stability of the frequency source. In particular, the use of cavities made from Ultra-Low-Expansion (ULE) glass, commonly used for atomic clocks [16], achieves effective thermal expansion coefficients smaller than $10^{-8} \mathrm{~K}^{-1}$ and their optical resonances provide an etalon to which the laser emission frequency can be locked [17]. To obtain the best results from optical feedback (down to below the theoretical $\mathrm{Hz}$ level) [18], a careful design of the cavity is mandatory [19]. Here, a special emphasis is placed on the estimation of the relative magnitudes of external disturbances to the overall cavity stability. Our goal is to thoroughly examine the design developed in [20] (see Supplementary Materials) and search for ways to obtain a high-end cavity which could be field deployable: compact and light enough to be carried, and stable enough to be usable outside the confines of a specialised laboratory. As the performances of the existing system are beyond our present measurement capabilities (see companion paper [14]), we characterised the frequency deviations of the present system in perturbed conditions to extrapolate with thermal and vibrational models, the actual performances of the cavity. We provide a description of the modelling approach, of the results compared to experimental values, as well as the implication for the use of such cavities in infrared spectroscopy for metrology and geosciences purposes. 


\section{Numerical approach}

The objective of the analysis effort was twofold: to decide whether it was possible to improve the existing design performance, and to determine which are the key factors limiting the stability of the system. The two major contributors first considered are: (M1) the quasi-static deformation due to fluctuations in environment temperature (thermal-mechanical effects), and (M2) the displacement of the mirrors due to bending induced by dynamic motion of the cavity (vibrational effects). A number of additional effects have been considered, for the sake of completeness: (m1) variable heat load on mirrors due to fluctuations in optical power stored in the cavity, (m2) variable heat load on mirrors due to external IR radiation transmitted by optical port windows, (m3) variable heat load on legs due to kinetic energy converted into heat via damping, (m4) unsteady vacuum refractive index, (m5) time-dependent force on mirrors due to unsteady radiation pressure and (m6) gravity effects due to tilt of the table.

Common practice to estimate the temperature propagation into an arbitrary system [Contributor (M1) and (m2)] is either to build condensed models [21] and analyse them using either approximate closed form solutions, or to use the transient analysis capabilities of conventional thermal packages. Approximate closed-form solutions make use of a series of lumped masses connected by heat links. Not only does this become cumbersome in situations where radiation is playing a key role, but also large inaccuracies are expected in cases where multiple enclosures are present, which is presently the case. However, the benefit of such a condensed model is that it can be processed at low computational costs, either in time or frequency domains. The second technique, thermal packages of transient calculations, can be applied as long as external temperature time series are available, but is computationally expensive, generates huge amounts of data and brings little insight into the physics of the system response. Lastly, some modal-based methods have in effect been developed [22], but the corresponding tools are and will remain proprietary (see for example [23])

Therefore, we implemented an approach solving all the aforementioned shortcomings. It involves working within a general-purpose Finite Elements Model (in our case: ANSYS Mechanical APDL, see Fig. 1), and extending its capabilities using its recently introduced matrix manipulation language (APDL Math). Specifically, two additional capabilities have been implemented: (1) thermal modal analysis and (2) thermal harmonic analysis (see Code 1-4, Supplementary Materials). These additional tools allow for rigorously deriving the thermal modes and the Thermal Transfer Function (TTF) of the system. Knowing the statistical properties of the external temperature fluctuations by means of their Power Spectral Density (PSD), it is possible to quantitatively estimate the

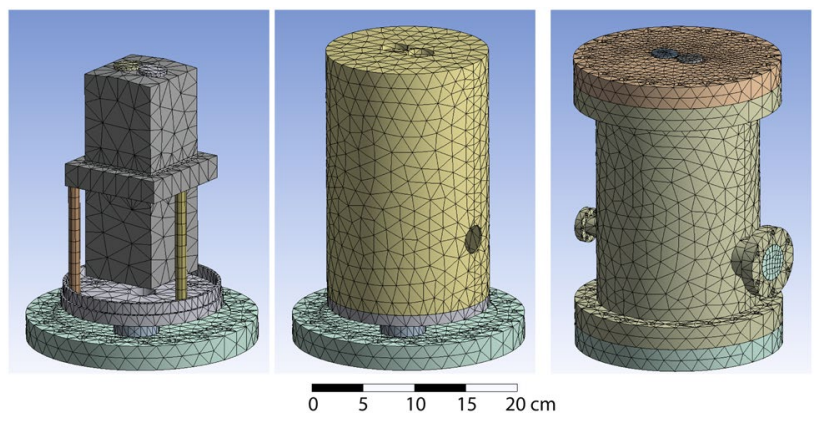

Fig. 1 Description of the FEM grid: from left to write, the cavity standing on the bottom flange, the inner shield and the vacuum chamber

temperature response properties. Lastly, the temperature fluctuations are converted into length variations by means of a thermo-mechanical analysis. The fractional length variation of the PSD can be used to derive typical indicators such as the Allan variance [24] of the cavity optical frequency in a realistically modelled environment.

The FEM accounts for both conduction and radiation heat transfer modes. Radiation is captured by computing view factors between each pair of radiating surface elements of the designated enclosures. Once this task is done, a superelement (i.e. a conductivity matrix) is added to the problem. In this way, the radiative heat transfer is linearised and matrix methods can be applied. In our case, all radiating surfaces are considered to be part of a single radiation enclosure, since there are openings connecting the inner and outer surfaces of the cavity, mirrors, inner shield, vacuum chamber, and the windows. Consequently, a single superelement is used, allowing to include all the "stray light" (IR radiation) radiated by Vacuum chamber and windows [contributor (m2)] directly onto the cavity and mirrors.

Once the model has been setup, it is useful to calculate the first thermal modes, as this helps understanding which heat paths are active, depending on the time scale of the external fluctuations. In our case, the first three thermal modes are displayed on Fig. 2. Clearly, the associated phenomena are as follows:

- Mode 1 ( $\tau=54651 \mathrm{~s})$ : cavity coming to equilibrium with vacuum chamber via Macor legs (conduction).

- Mode $2(\tau=3530 \mathrm{~s})$ : upper and lower ends of cavity coming to equilibrium with each other (diffusion).

- Mode 3 ( $\tau=3052 \mathrm{~s}$ ): inner thermal shield coming to equilibrium with vacuum chamber via Macor spacers (conduction).

From those modes, some physical insight can be gained, namely from mode 1 , there is a clear indication that the legs provide excellent insulation from thermal disturbances, 

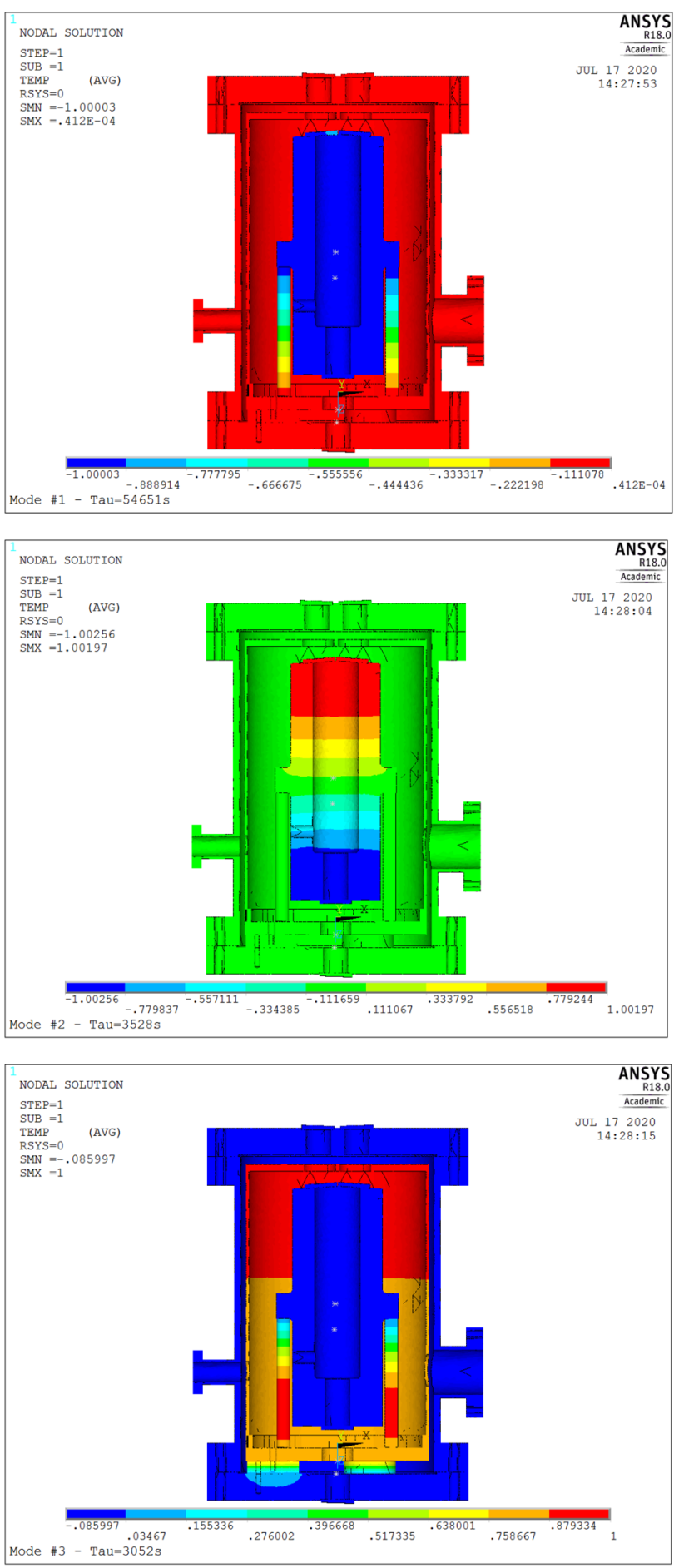

Fig. 2 First three thermal modes (normalized to unity)

since the first time constant is longer than half a day: daily temperature fluctuations (day/night cycle) will be attenuated by a factor equal to about $2 \pi \times 56400 / 86400 \simeq 4$ ). Examining mode 2, we know that upper and lower mirrors will not experience the same temperature for disturbances shorter than one hour. From mode 3, we deduce that the inner thermal shield only offers additional filtering effect for fluctuations shorter than about one hour. Clearly, if we want second order filtering, we could shorten the MACOR legs and use the gained space to lengthen the MACOR spacers.

The thermal frequency response was calculated in the frequency range from 0.1 to 1440 cycles per day, i.e. periods of temperature fluctuations ranging from 10 days to 1 minute, respectively. Excitation is provided by enforcing temperature variations on the outer surface of the vacuum chamber. The values selected for surface emissivities, which we believe are the major sources of uncertainty to the accuracy of the thermal transfer functions, are given on top of Fig. 2, and discussed in the supplementary material. Thermal response is evaluated by estimating the average temperature at a number of key locations: the surface of the inner vacuum chamber, the shield, the spacer and the three mirrors. The corresponding thermal transfer functions are plotted in Fig. 3.

For long periods, the thermal transfer function tends to unity, meaning that the thermal equilibrium is reached. In our case, the $3 \mathrm{~dB}$ cutoff frequency is $f \leq 0.3$ cycles per day, i.e. for cyclic temperature variations with periods longer than 3 days. In the range $0.3-3$ cycles per day, the responses of the spacer and the mirror are similar, and follow the expected $1 / f$ trend. At higher frequencies though, the responses of the spacer and the mirrors notably differ, both in terms of amplitude and phase, something that would not be taken into account by the classical lumped masses approach. While the spacer keeps following the same trend, the upper and lower mirrors exhibit a nearly flat response in the range $10^{1}-10^{2}$ cycles per day. This is due to a "thermal shunt" caused by radiation coming from the windows onto the mirrors [contribution (m2)]. Finally, at even higher frequencies, inertia from the mirrors takes over and induces a filtering effect $(1 / f)$. This complex behaviour exemplifies the additional physics that is predicted by the FEM approach but is hardly accounted for by the classical lumped masses approach (see Code 1-4, Supplementary Materials).

\section{Results}

We take advantage of the FEM approach to evaluate the variation in cavity length associated with the different contributors linked with temperature variations and vibrations. We first estimate the impact of change in temperature, using the structural FEM. The vertical displacement induced by a $1 \mathrm{~K}$ temperature step (from 300 to $301 \mathrm{~K}$ ) applied to both the lower and upper mirrors is plotted in Fig. 4. Both upper and lower mirrors induce cavity length changes that are larger than that of the spacer. This somewhat counter-intuitive phenomenon is induced by motion amplification due to mechanical bending of the mirrors, which have instantaneous coefficient of thermal expansion larger than that of Zerodur, are 

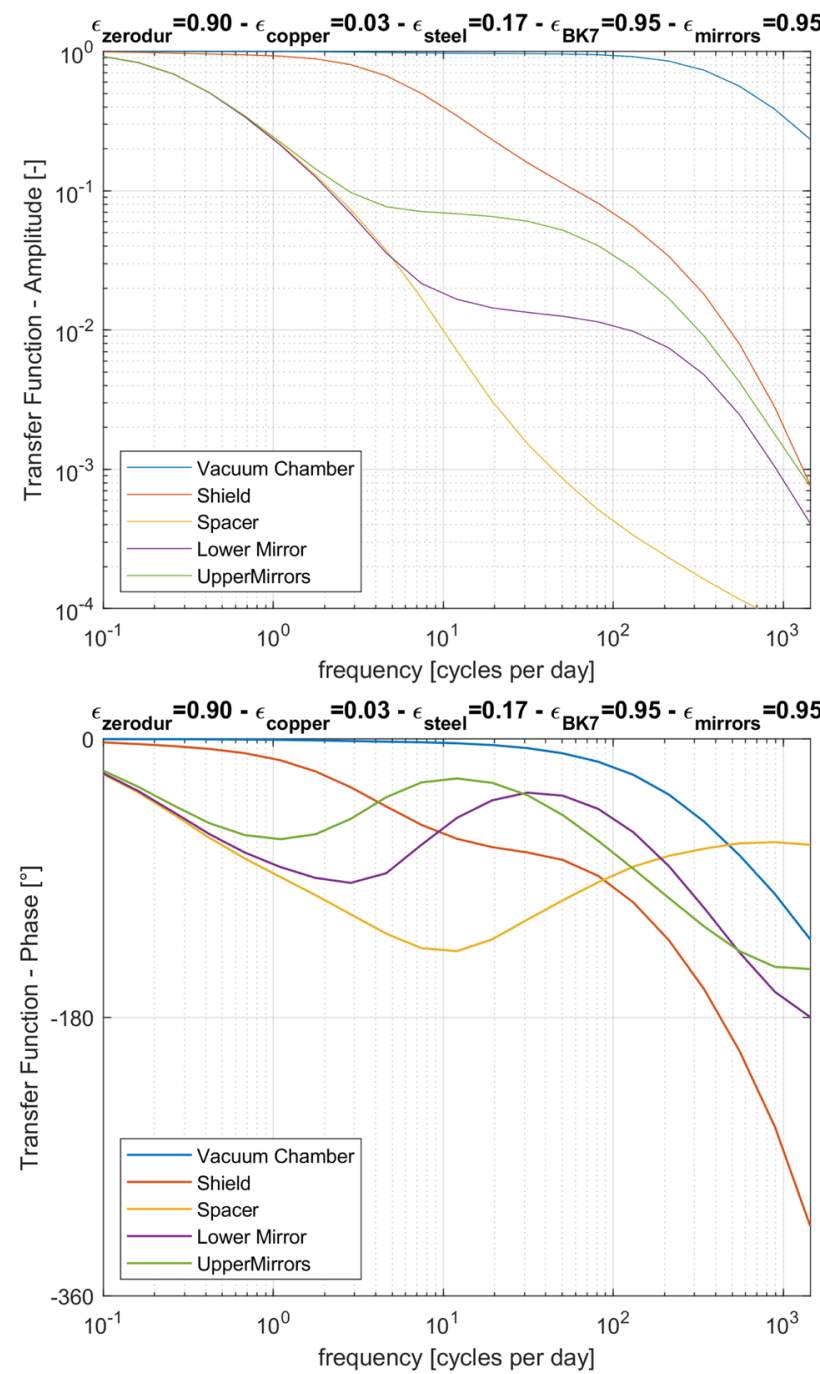

Fig. 3 Thermal transfer functions calculated by the model for the different components of the cavity. Top: amplitude Bot: phase

free to expand on one side, and blocked on the other. Note that at the interface between spacer and mirrors, we assumed perfect contact, i.e. no sliding nor uplift.

Numerically, the contributions of the mirrors to the cavity length variations are $8.5 \mathrm{~nm} \mathrm{~K}^{-1}$ for the lower mirror, and $9.0 \mathrm{~nm} \mathrm{~K}^{-1}$ for the upper mirrors. The corresponding fractional change cavity length per unit temperature reads: $4.6 \times 10^{-8} \mathrm{~K}^{-1}$ for the lower mirror and $4.9 \times 10^{-8} \mathrm{~K}^{-1}$ for each upper mirror. Accounting for the contribution of the spacer (that is, the instantaneous Coefficient of Thermal Expansion (CTE) of Zerodur, i.e. $1.0 \times 10^{-8} \mathrm{~K}^{-1}$ ) the total (equivalent) CTE for the entire cavity is about $10.4 \times 10^{-8}$ $\mathrm{K}^{-1}$, and is largely (about $90 \%$ ) dominated by the mirror contributions. In terms of frequency drift, one can infer an overall optical frequency change of about $22 \mathrm{MHz} \mathrm{K}^{-1}$, i.e. 11 times the increase expected for an ideal, homogeneous Zerodur cavity $\left(2.1 \mathrm{MHz} \mathrm{K}^{-1}\right)$. Experimentally, we tested this by changing the setpoint of the external thermal shield from 298 to $299 \mathrm{~K}$, resulting in an optical frequency shift of $11 \mathrm{MHz}$, i.e. half the anticipated value of $22 \mathrm{MHz}$ [14]. Nevertheless, this confirms the additional contribution from the mirrors. Also, and even more importantly, this experimental result sets the temperature stability limit to reach the $\mathrm{Hz} \mathrm{s}^{-1}$ criterion for optical frequency stability: the drift rate of the temperature within the cavity must be kept below $3.1 \times 10^{-8} \mathrm{~K} \mathrm{~s}^{-1}$.

The actual thermal excursions have been experimentally monitored on the outer surface of the vacuum chamber, which provided an input PSD of temperature variations (PSD in thick dark blue in Fig. 5). It is multiplied by the square of the frequency response functions (from Fig. 3) to yield the temperature response PSD of the cavity (Fig. 5), and in turn the standard deviations of its temperature fluctuation and fluctuation rate (Table 1).

We use the temperature fluctuation rates given in Table 1 to estimate the drift rate of the cavity using the actual temperature sensitivity of the cavity drift rate $\left(3.1 \times 10^{-8} \mathrm{~K} \mathrm{~s}^{-1}\right)$, obtained from the temperature step), and the temperature fluctuation rate. We observe a significant difference in thermal stability between the spacer and the mirrors (last three lines in Table 1). While the three of them exhibit comparable temperature deviation amplitudes, the associated drift rates are drastically different. Most importantly, the drift rate of the upper mirrors is more than 20 times higher than that of the spacer. This effect is further amplified by the larger thermo-mechanical susceptibility of the mirrors, such that the cavity optomechanical response is dominated by the mirrors. Quantitatively, the effective temperature drift rate, given by the combination of the upper and lower mirrors contributions is $0.11 \mu \mathrm{K} \mathrm{s}^{-1}$, that is, significantly above the previously defined threshold of $3.1 \times 10^{-8} \mathrm{~K} \mathrm{~s}^{-1}$. This predicts a drift of $3.5 \mathrm{~Hz} \mathrm{~s}^{-1}$, hence above the $1 \mathrm{~Hz} \mathrm{~s}^{-1}$ target, and in decent agreement (factor of 2 ) with the experimental $\operatorname{drift}\left(1.7 \mathrm{~Hz} \mathrm{~s}^{-1}\right.$, [14]).

We then evaluate the sensitivity of the system to vibrations [Contributor (M2)] by simulating the effect of a unitary $\left(1 \mathrm{~mm} \mathrm{~s}^{-2}\right)$ acceleration enforced at base. We use the outputs of the model to evaluate the corresponding change in the optical cavity length per unit of acceleration (Fig. 6, middle). In the quasi-static range (below $100 \mathrm{~Hz}$ ), the fractional length acceleration sensitivity is $4 \times 10^{-9}\left(\mathrm{~m} \mathrm{~s}^{-2}\right)^{-1}$ vertically, and $4 \times 10^{-10}\left(\mathrm{~m} \mathrm{~s}^{-2}\right)^{-1}$ horizontally (not shown). Above $100 \mathrm{~Hz}$, some resonant amplification occurs, because of the transverse flexibility of the cavity support legs. Assuming perfect contact at the interface, the upper mirrors themselves should exhibit a local bending mode at $5.7 \mathrm{kHz}$, out of the frequency range of our study.

To quantitatively compute the expected line width broadening induced in a realistic environnement, the accelerations at the outer boundary of the system were recorded and 
Fig. 4 Axial displacement (nm) of the mirrors for $1 \mathrm{~K}$ temperature increase relative to spacer: left lower mirror, right top mirrors

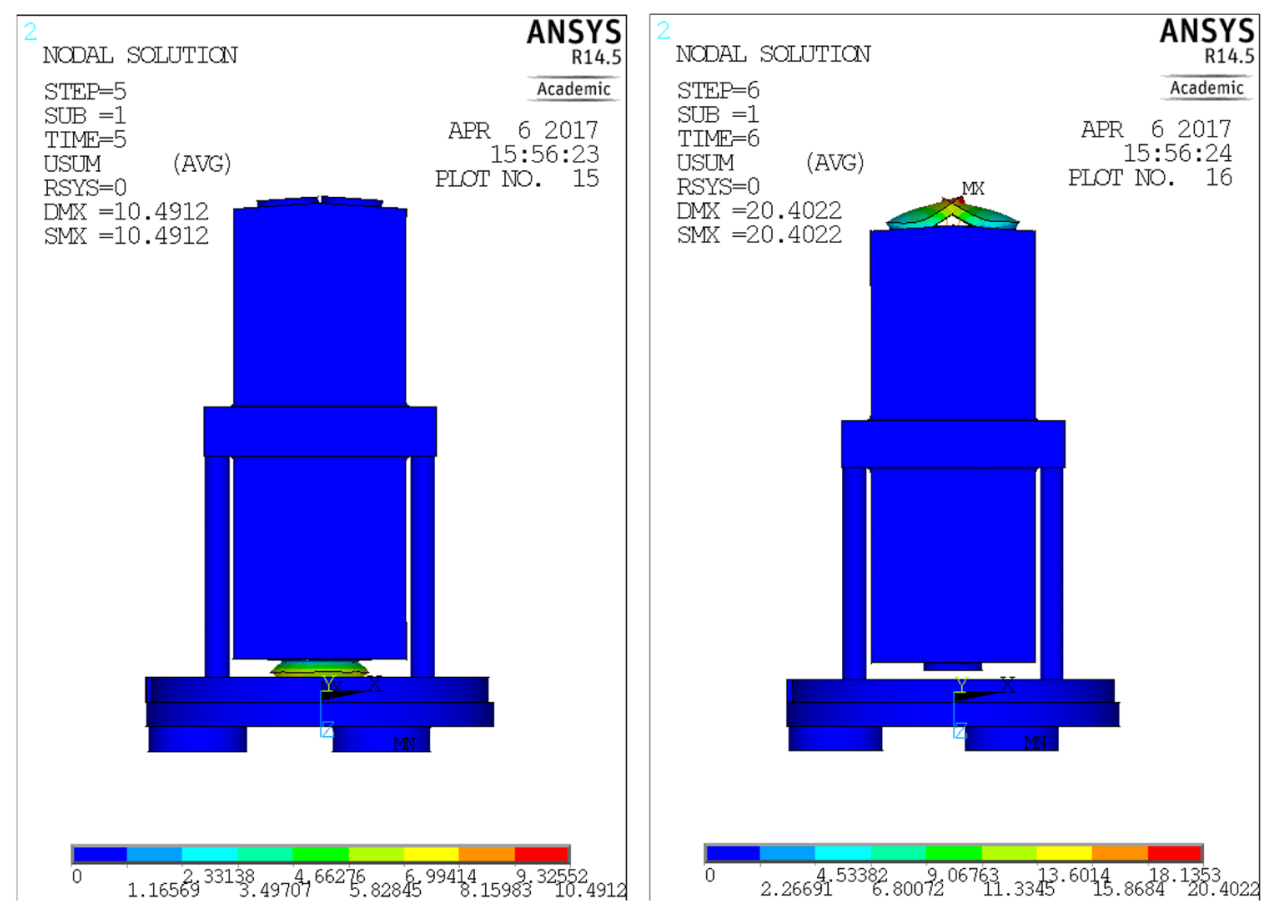

used as an input for the FEM approach. We recorded a time series of the acceleration of the outer surface of the vacuum chamber for an hour, using an accelerometer IEPE type DJB A21/V in association with a conditioning power supply model MMF M32 (×100), which offered the best compromise in terms of sensitivity/mass/self-noise. Importantly, the accelerometer itself has an upper cutting frequency (10\%) of $2 \mathrm{kHz}$, which limits the bandwidth of the analysis. Also, signal below $10 \mathrm{~Hz}$ was clearly dominated by electronic noise and could not be used. Also, only the axial acceleration could be obtained. The transverse vibrations could not be adequately measured in a sufficiently broad bandwidth. However, it is expected that the corresponding contribution should remain small compared to the axial motion, since (1) the entire system is resting on an optical table, the latter being extremely stiff with respect to in-plane motion and (2) the cavity sensitivity is also expected to be comparatively lower along transverse directions. In the frequency range from 10 to $2000 \mathrm{~Hz}$, the measured Root Mean Square (RMS) acceleration is $0.48 \pm 0.05 \mathrm{~mm} \mathrm{~s}^{-2}$ RMS (Fig. 6, upper plot). The FEM simulation is cumulated frequency-wise (see Supplementary Material, appendix E for mathematical definition of cumulated length variation) and predicts an RMS change in cavity length of 0.72 pm (orange curve in Fig. 6), in reasonably close agreement with the quasi-static approach $(0.48 \mathrm{pm})$, showing that our current design induces limited amplification of the environmental mechanical noise. These results can be transferred into an estimate of the frequency stability of the cavity. These are presented as an Allan Variance frequency deviation plot in Fig. 7. Typically, for $1 \mathrm{~ms}$

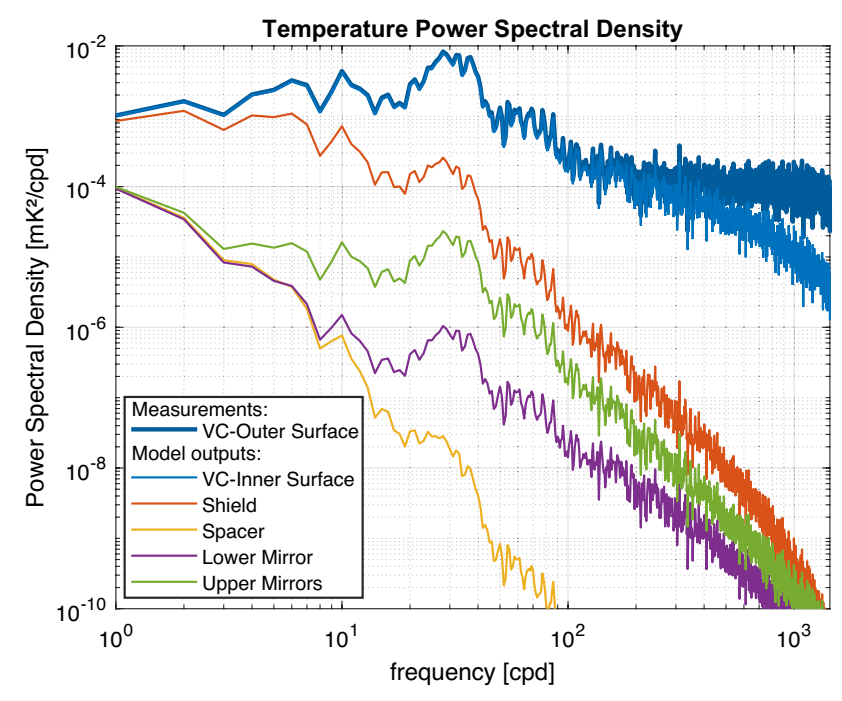

Fig. 5 Temperature PSDs for the different component of the system. The Vacuum chamber outer surface temperature PSD has been used as an input, whereas all the others are generated by the FEM

Table 1 Standard deviations of temperature fluctuations $\sigma_{T}$ and fluctuation rate $\sigma_{T^{\prime}}$

\begin{tabular}{lll}
\hline Location & $\sigma_{T}[\mu \mathrm{K}]$ & $\sigma_{T^{\prime}}\left[\mu \mathrm{K} \mathrm{s}^{-1}\right]$ \\
\hline VC-outer surface & 590 & 22.4 \\
VC-inner surface & 496 & 8.52 \\
Shield & 117 & 0.21 \\
Spacer & 18 & 0.004 \\
Lower mirror & 18 & 0.032 \\
Upper mirrors & 29 & 0.082 \\
\hline
\end{tabular}



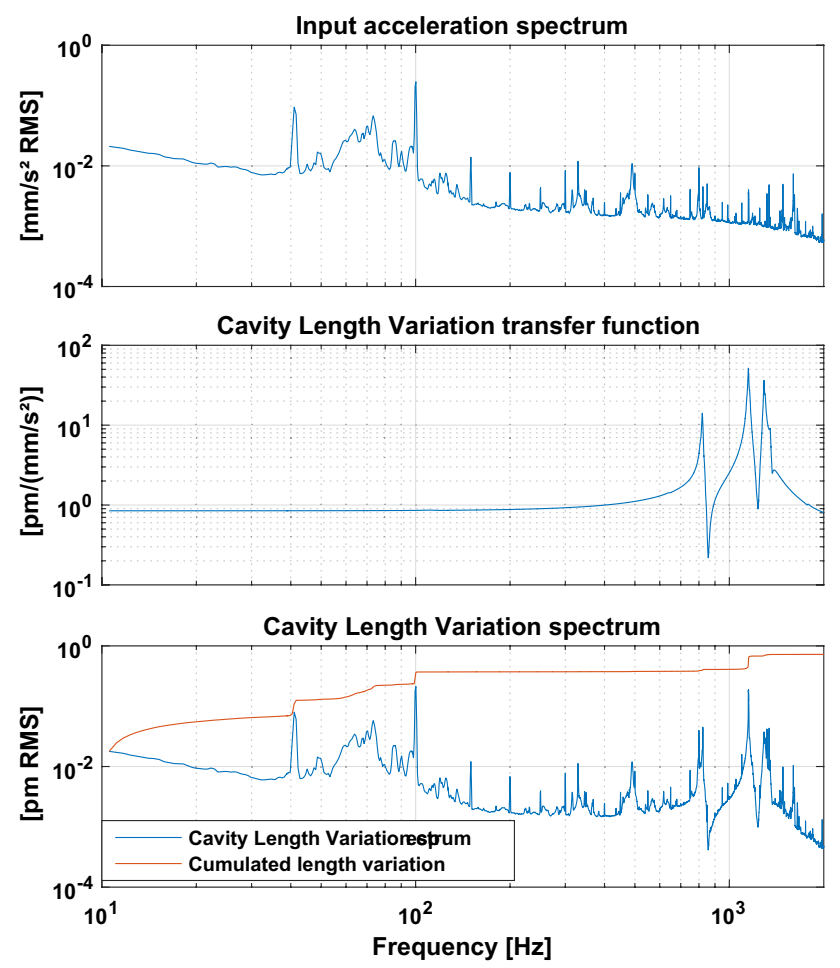

Fig. 6 Vibrational frequency response of the cavity: top: typical input acceleration spectrum measured by the accelerometer fixed on the cavity outer shell, middle: transfer function obtained by the FEM, and bottom: spectrum of the cavity length variations

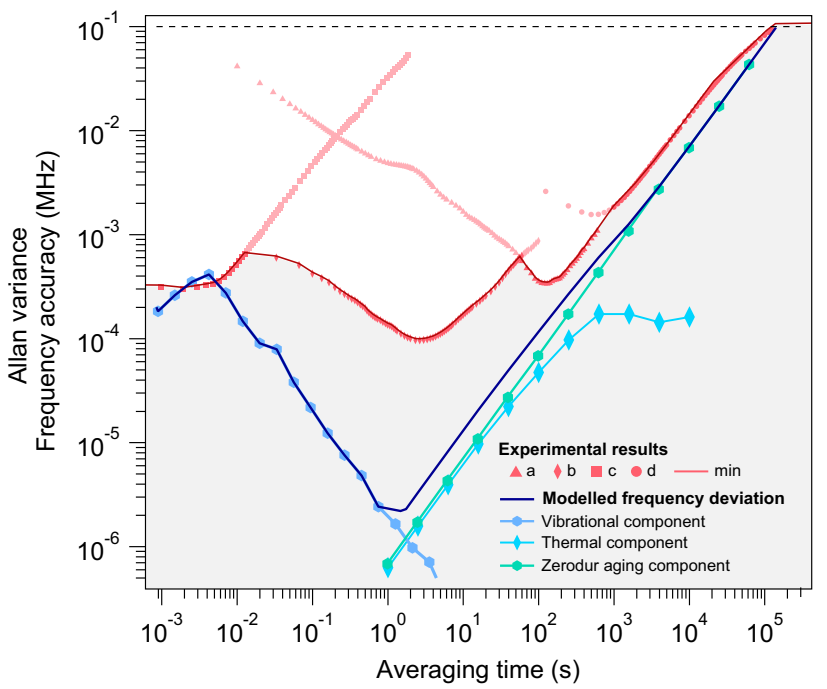

Fig. 7 Simulated and observed characterisation of the frequency deviation of the cavity: red dots are observations from various methods, see Companion Paper [14], where the low opacity points represent excess noise due to the measurement technique, the grey area corresponds to the range of values that could be associated with the drift according to observations; blue dots and line are the simulations (hexagons: vibrational, diamonds: thermal and circle: ageing) time scale, we obtained a frequency stability of $190 \mathrm{~Hz}$, in close agreement with short-term observations $(181 \mathrm{~Hz})$ [14].

\section{Discussion}

Overall, we were able to obtain the power spectral density of the changes in length of the cavity induced by the main contributors: for frequencies below $1 \mathrm{~Hz}$, the thermo-mechanical processes [contributors (M1) and (m2)], and for frequencies above $1 \mathrm{~Hz}$, the vibrations [contributor (M2)]. For low frequency, the modelled and measured drift rate are in approximate agreement ( 3.4 and $1.7 \mathrm{~Hz} \mathrm{~s}^{-1}$, respectively). This drift rate is acceptable for IR field spectrometry $[4,5]$, for which a precision of $1 \mathrm{kHz}$ on the frequency axis can still provide satisfactory precision [7], and can be achieved by hourly referencing the frequency of the laser on a very fine spectroscopic pattern of a Lamb-dip [13]. However, for stricter applications such as metrology, drift rates below $1 \mathrm{~Hz} \mathrm{~s}^{-1}$ are needed. The FEM approach enables to determine that the mirrors are the main contributors. We could verify numerically that a Zerodur ring on the back of the mirrors would prevent the deformations [17]. In general, for both vibrational and thermal aspects, the only partially supported mirrors solution, if economically beneficial, limits the performances compared to more compact and fully contacted designs [25].

We converted the different results into Allan Variance of the frequency deviation of the cavity. We additionally included the impact of the cavity ageing using long-term drift rate obtained by measuring the frequency deviation of the cavity over 2 years [14]. We observe good agreement between modelling approach and observations at small time scales (around $1 \mathrm{~ms}$ ) and at long time scales (drift, larger than $100 \mathrm{~s}$ ). In the frequency range around $1 \mathrm{~Hz}$, the modelling approach yields significantly better performances than the observations, probably due to experimental limits to determine the true performances of the laser source [14]. On average, the drift rate of the cavity is around $0.7 \mathrm{~Hz} \mathrm{~s}^{-1}$, typical for a Zerodur cavity, but larger than an ULE cavity [26, 27]. From the thermomechanical models, we expect that the performances of the cavity are better than our present measurement capabilities (red points in Fig. 7), in particular for around $1 \mathrm{~Hz}$ where precision and accuracy of $1 \mathrm{~Hz}$ would be required.

\section{Conclusion}

In conclusion, we simulated the thermo-mechanical and vibrational behaviour of a $\mathrm{V}$-shaped optical feedback cavity with demonstrated $\mathrm{Hz}$ level stability ( $2 \mathrm{~Hz} \mathrm{~s}^{-1}$ drift rate) and sub$\mathrm{kHz}$ emission linewidth at $215 \mathrm{THz}$. The high performances of the cavity limited the ability to evaluate its frequency linewidth and drift rate in normal operation conditions. We 
had to generate perturbed environmental conditions to observe significant frequency deviations, and be able to validate the model, and thus extrapolate the performances of the cavity in stable conditions, which the instrument faces in normal operations. A specific thermo-mechanical analysis procedure was developed, allowing for the capture of dynamic heat transfer mechanisms with high fidelity. The numerical results obtained compared favourably against experimental data in perturbed and stable conditions. It was found that in our case, the local temperature fluctuations and the local bending of the mirrors were responsible for the vast majority of the drift and even more importantly of the drift rate. This suggests that local improvements, such as adding local (reflective) coating on the edges of the mirrors or contacting Zerodur rings on the back of the mirrors, would drastically improve the system stability. Also, a more compact design might be feasible, resulting in total weight reduction (the vacuum chamber representing over $75 \%$ of the total weight). More generally, this suite of predictive tools allows for the improvement of future generations of such reference cavities, either to improve performance or, as is particularly relevant here, to achieve a fully field deployable, rugged cavity design. Our short-term goal consists of designing a mechanically robust system that would be stable enough to serve as a calibration-free light source hosted in a field deployable high-end trace gas spectrometer. This requires the type of predictive tools presented here, providing quantitative insight into the influence of key design parameters.

Supplementary Information The online version contains supplementary material available at https://doi.org/10.1007/s00340-022-07779-x.

Funding Open Access funding enabled and organized by Projekt DEAL. The research leading to these results received funding from the European Research Council under the European Union's Seventh Framework Programme (FP7/2007-2013)/RC grant agreement number 306045, and from the Alexander von Humboldt fundation project DEAPICE.

Open Access This article is licensed under a Creative Commons Attribution 4.0 International License, which permits use, sharing, adaptation, distribution and reproduction in any medium or format, as long as you give appropriate credit to the original author(s) and the source, provide a link to the Creative Commons licence, and indicate if changes were made. The images or other third party material in this article are included in the article's Creative Commons licence, unless indicated otherwise in a credit line to the material. If material is not included in the article's Creative Commons licence and your intended use is not permitted by statutory regulation or exceeds the permitted use, you will need to obtain permission directly from the copyright holder. To view a copy of this licence, visit http://creativecommons.org/licenses/by/4.0/.

\section{References}

1. P. Werle, Spectrochim. Acta Part A Mol. Biomol. Spectrosc. 54(2), 197 (1998)

2. E. Kerstel, L. Gianfrani, Appl. Phys. B 92(3), 439 (2008)
3. E.J. Steig, V. Gkinis, A.J. Schauer, S.W. Schoenemann, K. Samek, J. Hoffnagle, K.J. Dennis, S.M. Tan, Atmos. Meas. Tech. 7(8), 2421 (2014). https://doi.org/10.5194/amt-7-2421-2014

4. J. Landsberg, D. Romanini, E. Kerstel, Opt. Lett. 39(7), 1795 (2014). https://doi.org/10.1364/OL.39.001795

5. T. Stoltmann, M. Casado, M. Daëron, A. Landais, S. Kassi, Anal. Chem. 89(19), 10129 (2017)

6. S. Kassi, A. Campargue, J. Chem. Phys. (2012). https://doi.org/ 10.1063/1.4769974

7. J. Burkart, T. Sala, D. Romanini, M. Marangoni, A. Campargue, S. Kassi, J. Chem. Phys. 142(19), 191103 (2015)

8. M.D. Ellehoj, H.C. Steen-Larsen, S.J. Johnsen, M.B. Madsen, Rapid Commun. Mass Spectrom. 27(19), 2149 (2013). https:// doi.org/10.1002/rcm.6668

9. M. Casado, A. Cauquoin, A. Landais, D. Israel, A. Orsi, E. Pangui, J. Landsberg, E. Kerstel, F. Prie, J.F. Doussin, Geochimica et Cosmochimica Acta 174, 54 (2016). https://doi.org/10.1016/j. gca.2015.11.009

10. M. Casado, A. Landais, V. Masson-Delmotte, C. Genthon, E. Kerstel, S. Kassi, L. Arnaud, G. Picard, F. Prie, O. Cattani, H.C. Steen-Larsen, E. Vignon, P. Cermak, Atmos. Chem. Phys. 16(13), 8521 (2016). https://doi.org/10.5194/acp-16-8521-2016

11. F. Ritter, H.C. Steen-Larsen, M. Werner, V. Masson-Delmotte, A. Orsi, M. Behrens, G. Birnbaum, J. Freitag, C. Risi, S. Kipfstuhl, Cryosphere 2016, 1 (2016). https://doi.org/10.5194/tc-2016-4

12. J. Burkart, D. Romanini, S. Kassi, Opt. Lett. 38(12), 2062 (2013)

13. S. Kassi, T. Stoltmann, M. Casado, M. Daëron, A. Campargue, J. Chem. Phys. 148(5), 54201 (2018). https://doi.org/10.1063/1. 5010957

14. M. Casado, T. Stoltmann, A. Landais, N. Jobert, M. Daëron, F. Prié, S. Kassi, Appl. Phys. B 128(3), 1-7 (2022)

15. B. Dahmani, L. Hollberg, R. Drullinger, Opt. Lett. 12(11), 876 (1987)

16. Y.Y. Jiang, A.D. Ludlow, N.D. Lemke, R.W. Fox, J.A. Sherman, L.S. Ma, C.W. Oates, Nat. Photon. 5(3), 158 (2011)

17. T. Legero, T. Kessler, U. Sterr, JOSA B 27(5), 914 (2010)

18. J. Alnis, A. Matveev, N. Kolachevsky, T. Udem, T.W. Hänsch, Phys. Rev. A 77(5), 53809 (2008). https://doi.org/10.1103/PhysR evA.77.053809

19. J. Millo, D.V. Magalhaes, C. Mandache, Y. Le Coq, E.M.L. English, P.G. Westergaard, J. Lodewyck, S. Bize, P. Lemonde, G. Santarelli, Phys. Rev. A 79(5), 53829 (2009)

20. M. Casado, Water stable isotopic composition on the East Antarctic Plateau: measurements at low temperature of the vapour composition, utilisation as an atmospheric tracer and implication for paleoclimate studies. Ph.D. thesis, Paris Saclay (2016)

21. X. Dai, Y. Jiang, C. Hang, Z. Bi, L. Ma, Opt. Express 23(4), 5134 (2015)

22. T.M. Shih, J.T. Skladany, Numer. Heat Transf. 6(4), 409 (1983). https://doi.org/10.1080/01495728308963097

23. J.W. Palmieri, Cave 3-a general transient heat transfer computer code utilizing eigenvectors and eigenvalues. Technical report (NASA, 1978)

24. D.W. Allan, Proc. IEEE 54(2), 221 (1966)

25. L. Chen, J.L. Hall, J. Ye, T. Yang, E. Zang, T. Li, Phys. Rev. A 74(5), 53801 (2006)

26. P. Dubé, A.A. Madej, J.E. Bernard, L. Marmet, A.D. Shiner, Appl. Phys. B 95(1), 43 (2009)

27. H. Bach, Low Thermal Expansion Glass Ceramics (Springer, Berlin, 2013)

Publisher's Note Springer Nature remains neutral with regard to jurisdictional claims in published maps and institutional affiliations. 